\title{
Intersection Curves of Implicit and Parametric Surfaces in $\mathbb{R}^{3}$
}

\author{
Mohamed Abdel-Latif Soliman, Nassar Hassan Abdel-All, \\ Soad Ali Hassan, Sayed Abdel-Naeim Badr \\ Department of Mathematics, Faculty of Science, Assiut University, Assiut, Egypt \\ E-mail: sayed_badr@ymail.com \\ Received June 9, 2011; revised June 30, 2011; accepted July 7, 2011
}

\begin{abstract}
We present algorithms for computing the differential geometry properties of Frenet apparatus $(\mathrm{t}, \mathrm{n}, \mathrm{b}, \kappa, \tau)$ and higher-order derivatives of intersection curves of implicit and parametric surfaces in $\mathbb{R}^{3}$ for transversal and tangential intersection. This work is considered as a continuation to Ye and Maekawa [1]. We obtain a classification of the singularities on the intersection curve. Some examples are given and plotted.
\end{abstract}

Keywords: Geometric Properties, Frenet Frame, Frenet Apparatus, Frenet-Serret Formulas, Surface-Surface Intersection, Transversal Intersection, Tangential Intersection, Dupin Indicatrices

\section{Introduction}

The intersection problem is a fundamental process needed in modeling complex shapes in CAD/CAM system. It is useful in the representation of the design of complex objects, in computer animation and in $\mathrm{NC}$ machining for trimming off the region bounded by the self-intersection curves of offset surfaces. It is also essential to Boolean operations necessary in the creation of boundary representation in solid modeling [1]. The numerical marching method is the most widely used method for computing intersection curves in $\mathbb{R}^{3}$. The Marching method involves generation of sequences of points of an intersection curve in the direction prescribed by the local differential geometry [2,3]. Willmore [4] described how to obtain the unit tangent, the unit principal normal, the unit binormal, the curvature and the torsion of the transversal intersection curve of two implicit surfaces [5]. Kruppa [6] explained that the tangential direction of the intersection curve at a tangential intersection point corresponds to the direction from the intersection point towards the intersection of the Dupin indicatrices of the two surfaces. Hartmann [7] provided formulas for computing the curvature of the transversal intersection curves for all types of intersection problems in Euclidean 2-space. Kriezis et al. [8] determined the marching direction for tangential intersection curves based on the fact that the determinant of the Hessian matrix of the oriented distance function is zero. Luo et al. [9] presented a method to trace such tangential intersection curves for parametric-parametric surfaces employing the marching method. The marching direction is obtained by solving an undetermined system based on the equilibrium of the differentiation of the two normal vectors and the projection of the Taylor expansion of the two surfaces onto the normal vector at the intersection point. Ye and Maekawa [1] presented algorithms for computing all the differential geometry properties of both transversal and tangentially intersection curves of two parametric surfaces. They described how to obtain these properties for two implicit surfaces or parametric-implicit surfaces. They also gave algorithms to evaluate the higher-order derivative of the intersection curves. Aléssio [10] studied the differential geometry properties of intersection curves of three implicit surfaces in $\mathbb{R}^{4}$ for transversal intersection, using the implicit function theorem.

In this study, we present algorithms for computing the deferential geometry properties of both transversal and tangentially intersection curves of implicit and Parametric surfaces in $\mathbb{R}^{3}$ as an extension to the works of [1].

This paper is organized as follows: Section 2 briefly introduces some notations, definitions and reviews of differential geometry properties of curves and surfaces in $\mathbb{R}^{3}$. Section 3 derives the formulas to compute the properties for the transversal intersection. Section 4 derives the formulas to compute the properties for the tangential intersection. Some examples of transversal and tangentially intersection are given and plotted in Section 5. Finally, conclusion is given in Section 6. 


\section{Geometric Preliminaries [1, 11-13]}

Let us first introduce some notation and definitions. The scalar product and cross product of two vectors $\boldsymbol{a}$ and $\boldsymbol{c}$ are expressed as $\langle\boldsymbol{a}, \boldsymbol{c}\rangle$ and $\boldsymbol{a} \times \boldsymbol{c}$, respectively. The length of the vector $\boldsymbol{a}$ is $\|\boldsymbol{a}\|=\sqrt{\langle\boldsymbol{a}, \boldsymbol{a}\rangle}$.

\subsection{Differential Geometry of the Curves in $\mathbb{R}^{3}$}

Let $\alpha: I \rightarrow \mathbb{R}^{3}$ be a regular curve in $\mathbb{R}^{3}$ with arc-length parameterization,

$$
\boldsymbol{\alpha}(s)=\left(x_{1}(s), x_{2}(s), x_{3}(s)\right)
$$

The notation for the differentiation of the curve $\alpha$ in relation to the arc length $s$ is $\boldsymbol{\alpha}^{\prime}(s)=\frac{\mathrm{d} \alpha}{\mathrm{d} s}, \quad \boldsymbol{\alpha}^{\prime \prime}(s)=\frac{\mathrm{d}^{2} \alpha}{\mathrm{d} s^{2}}$, $\boldsymbol{\alpha}^{\prime \prime \prime}(s)=\frac{\mathrm{d}^{3} \alpha}{\mathrm{d} s^{3}}$. Then from elementary differential geometry, we have

$$
\begin{gathered}
\boldsymbol{\alpha}^{\prime}(s)=\boldsymbol{t} \\
\boldsymbol{\alpha}^{\prime \prime}(s)=\kappa \boldsymbol{n} \\
\kappa^{2}(s)=\left\langle\boldsymbol{\alpha}^{\prime \prime}, \boldsymbol{\alpha}^{\prime \prime}\right\rangle
\end{gathered}
$$

where $\boldsymbol{t}$ is the unit tangent vector field and $\boldsymbol{\alpha}^{\prime \prime}$ is the curvature vector. The factor $\kappa$ is the curvature and $\boldsymbol{n}$ is the unit principal normal vector. The unit binormal vector $\boldsymbol{b}$ is defined as

$$
\boldsymbol{b}(s)=\boldsymbol{t} \times \boldsymbol{n}
$$

The vectors $\boldsymbol{t}, \boldsymbol{n}, \boldsymbol{b}$, are called collectively the FrenetSerret frame. The Frenet-Serret formulas along $\boldsymbol{\alpha}$ are given by

$$
\begin{aligned}
& \boldsymbol{t}^{\prime}(s)=\kappa \boldsymbol{n}, \\
& \boldsymbol{n}^{\prime}(s)=-\kappa \boldsymbol{t}+\tau \boldsymbol{b}, \\
& \boldsymbol{b}^{\prime}(s)=-\tau \boldsymbol{n} .
\end{aligned}
$$

where $\tau$ is the torsion which is given by

$$
\tau=\frac{\left\langle\boldsymbol{b}, \boldsymbol{\alpha}^{\prime \prime \prime}\right\rangle}{\kappa}
$$

provided that the curvature does not vanish.

\subsection{Differential Geometry of the Parametric Surfaces in $\mathbb{R}^{3}$}

Assume that $\boldsymbol{R}\left(u_{1}, v_{2}\right)$ is a regular parametric surface. In other words $\boldsymbol{R}_{1} \times \boldsymbol{R}_{2} \neq 0$, where $\boldsymbol{R}_{r}=\frac{\partial \boldsymbol{R}}{\partial u_{r}}(r=1,2)$ denote to partial derivatives of the surface $R$. The unit sur- face normal vector field of the surface $\boldsymbol{R}$ is given by

$$
\boldsymbol{N}=\frac{\boldsymbol{R}_{1} \times \boldsymbol{R}_{2}}{\left\|\boldsymbol{R}_{1} \times \boldsymbol{R}_{2}\right\|}
$$

The first fundamental form coefficients of the surface $\boldsymbol{R}$ are given by

$$
g_{p q}=\left\langle\boldsymbol{R}_{p}, \boldsymbol{R}_{q}\right\rangle ; p, q=1,2
$$

The second fundamental form coefficients of the surface $\boldsymbol{R}$ are given by

$$
L_{11}=\left\langle\boldsymbol{R}_{11}, \boldsymbol{N}\right\rangle, L_{12}=\left\langle\boldsymbol{R}_{12}, \boldsymbol{N}\right\rangle, L_{22}=\left\langle\boldsymbol{R}_{22}, \boldsymbol{N}\right\rangle
$$

Let $u_{r}(s), r=1,2$ in the $u_{1} u_{2}$-plane defines a curve on the surface $\boldsymbol{R}$ which can be written as

$$
\boldsymbol{\alpha}(s)=\boldsymbol{R}\left(u_{1}(s), u_{2}(s)\right)
$$

Then the three derivatives of the curve $\boldsymbol{\alpha}$ are given by

$$
\begin{aligned}
\boldsymbol{\alpha}^{\prime}=\boldsymbol{R}_{1} u_{1}^{\prime}+\boldsymbol{R}_{2} u_{2}^{\prime} \\
\boldsymbol{\alpha}^{\prime \prime}=\boldsymbol{R}_{11}\left(u_{1}^{\prime}\right)^{2}+2 \boldsymbol{R}_{12} u_{1}^{\prime} u_{2}^{\prime}+\boldsymbol{R}_{22}\left(u_{2}^{\prime}\right)^{2}+\boldsymbol{R}_{1} u_{1}^{\prime \prime}+\boldsymbol{R}_{2} u_{2}^{\prime \prime} \\
\boldsymbol{\alpha}^{\prime \prime \prime}=\boldsymbol{R}_{1} u_{1}^{\prime \prime \prime}+\boldsymbol{R}_{2} u_{2}^{\prime \prime \prime}+\boldsymbol{R}_{111}\left(u_{1}^{\prime}\right)^{3}+\boldsymbol{R}_{222}\left(u_{2}^{\prime}\right)^{3} \\
+3\left(\boldsymbol{R}_{11} u_{1}^{\prime} u_{1}^{\prime \prime}+\boldsymbol{R}_{12}\left(u_{1}^{\prime \prime} u_{2}^{\prime}+u_{1}^{\prime} u_{2}^{\prime \prime}\right)+\boldsymbol{R}_{22} u_{2}^{\prime} u_{2}^{\prime \prime}\right) \\
\\
+3 \boldsymbol{R}_{112}\left(u_{1}^{\prime}\right)^{2} u_{2}^{\prime}+3 \boldsymbol{R}_{122} u_{1}^{\prime}\left(u_{2}^{\prime}\right)^{2}
\end{aligned}
$$

The projection of the curvature vector $\boldsymbol{\alpha}^{\prime \prime}$ onto the unit normal vector field of the surface $\boldsymbol{R}$ is given by

$$
\left\langle\boldsymbol{\alpha}^{\prime \prime}, \frac{\boldsymbol{R}_{1} \times \boldsymbol{R}_{2}}{\left\|\boldsymbol{R}_{1} \times \boldsymbol{R}_{2}\right\|}\right\rangle=L_{11}\left(u_{1}^{\prime}\right)^{2}+2 L_{12} u_{1}^{\prime} u_{2}^{\prime}+L_{22}\left(u_{2}^{\prime}\right)^{2}
$$

\subsection{Differential Geometry of the Implicit Surfaces in $\mathbb{R}^{3}$}

Assume that $f\left(x_{1}, x_{2}, x_{3}\right)=0$ is a regular implicit surface. In other words $\nabla \boldsymbol{f} \neq 0$, where $\nabla \boldsymbol{f}=\left(f_{1}, f_{2}, f_{3}\right)$ is the gradient vector of the surface $f, f_{p}=\frac{\partial f}{\partial x_{p}}$, then the unit surface normal vector field of the surface $f$ is given by

$$
\boldsymbol{N}=\frac{\nabla \boldsymbol{f}}{\|\nabla \boldsymbol{f}\|}
$$

Let

$$
\boldsymbol{a}(s)=\left(x_{1}(s), x_{2}(s), x_{3}(s)\right)
$$

be a curve on the surface $f$ with constraint $f\left(x_{1}, x_{2}, x_{3}\right)=0$ then we have 


$$
\begin{gathered}
\boldsymbol{\alpha}^{\prime}=\left(x_{1}^{\prime}, x_{2}^{\prime}, x_{3}^{\prime}\right), \\
\boldsymbol{\alpha}^{\prime \prime}=\left(x_{1}^{\prime \prime}, x_{2}^{\prime \prime}, x_{3}^{\prime \prime}\right), \\
\boldsymbol{\alpha}^{\prime \prime \prime}=\left(x_{1}^{\prime \prime \prime}, x_{2}^{\prime \prime \prime}, x_{3}^{\prime \prime \prime}\right) . \\
\frac{\mathrm{d} f}{\mathrm{~d} s}=f_{1} x_{1}^{\prime}+f_{2} x_{2}^{\prime}+f_{3} x_{3}^{\prime}=0 \\
\frac{\mathrm{d}^{2} f}{\mathrm{~d} s^{2}}=f_{11}\left(x_{1}^{\prime}\right)^{2}+f_{22}\left(x_{2}^{\prime}\right)^{2}+f_{33}\left(x_{3}^{\prime}\right)^{2} \\
+2\left(f_{12} x_{1}^{\prime} x_{2}^{\prime}+f_{13} x_{1}^{\prime} x_{3}^{\prime}+f_{23} x_{2}^{\prime} x_{3}^{\prime}\right) \\
+f_{1} x_{1}^{\prime \prime}+f_{2} x_{2}^{\prime \prime}+f_{3} x_{3}^{\prime \prime}=0
\end{gathered}
$$

The projection of the curvature vector $\boldsymbol{\alpha}^{\prime \prime}$ onto the unit normal vector field of the surface $f$ is given by

$$
\left\langle\boldsymbol{\alpha}^{\prime \prime}, \frac{\nabla \boldsymbol{f}}{\|\nabla \boldsymbol{f}\|}\right\rangle=\frac{-\eta}{\sqrt{f_{1}^{2}+f_{2}^{2}+f_{3}^{2}}}
$$

where

$$
\begin{aligned}
\eta & =f_{11}\left(x_{1}^{\prime}\right)^{2}+f_{22}\left(x_{2}^{\prime}\right)^{2}+f_{33}\left(x_{3}^{\prime}\right)^{2} \\
& +2\left(f_{12} x_{1}^{\prime} x_{2}^{\prime}+f_{13} x_{1}^{\prime} x_{3}^{\prime}+f_{23} x_{2}^{\prime} x_{3}^{\prime}\right)
\end{aligned}
$$

\section{Transversal Intersection Curves}

Consider the intersecting implicit and parametric surfaces $f\left(x_{1}, x_{2}, x_{3}\right)=0$ and $\boldsymbol{R}=R\left(u_{1}, u_{2}\right) ; \quad \mathrm{c}_{1}<u_{1}<c_{2}$, $c_{3}<u_{2}<c_{4}$ such that, $f \neq 0, \boldsymbol{R}_{1} \times \boldsymbol{R}_{2} \neq 0$. Then the intersection curve of these surfaces can be viewed as a curve on both surfaces as

$$
\begin{gathered}
\boldsymbol{\alpha}(s)=\left(x_{1}(\mathrm{~s}), x_{2}(\mathrm{~s}), x_{3}(\mathrm{~s})\right) ; \quad f\left(x_{1}, x_{2}, x_{3}\right)=0, \\
\boldsymbol{\alpha}(s)=\boldsymbol{R}\left(u_{1}(\mathrm{~s}), u_{2}(\mathrm{~s})\right) ; \quad c_{1}<u_{1}<c_{2}, c_{3}<u_{2}<c_{4} .
\end{gathered}
$$

Then we have

$$
\boldsymbol{x}_{i}(s)=\boldsymbol{R}^{i}\left(\boldsymbol{u}_{1}(\mathrm{~s}), \boldsymbol{u}_{2}(\mathrm{~s})\right), \boldsymbol{i}=1,2,3
$$

where $\boldsymbol{R}\left(u_{1}(\mathrm{~s}), u_{2}(\mathrm{~s})\right)=\left(R^{1}, R^{2}, R^{3}\right)$. Then the surface $f$ can be expressed as

$$
h\left(u_{1}, u_{2}\right)=f\left(R^{1}, R^{2}, R^{3}\right)=0
$$

Thus the intersection curve is given by

$$
\begin{gathered}
\boldsymbol{\alpha}(s)=\boldsymbol{R}\left(u_{1}(\mathrm{~s}), u_{2}(\mathrm{~s})\right) ; h\left(u_{1}, u_{2}\right)=0, \\
c_{1}<u_{1}<c_{2}, c_{3}<u_{2}<c_{4}
\end{gathered}
$$

\subsection{Tangential Direction}

Differentiation (3.1) yields

$$
h_{1} u_{1}^{\prime}+h_{2} u_{2}^{\prime}=0
$$

where $h_{i}=\frac{\partial h}{\partial u_{i}}$, then we have

$$
u_{2}^{\prime}=\frac{-h_{1}}{h_{2}} u_{1}^{\prime}, \quad h_{2} \neq 0
$$

Since $\boldsymbol{\alpha}^{\prime}$ is the unit tangent vector field of the curve (3.2), then we have

$$
\left\|\boldsymbol{\alpha}^{\prime}\right\|=\left\langle\boldsymbol{R}_{1} u_{1}^{\prime} \boldsymbol{R}_{2} u_{2}^{\prime}, \boldsymbol{R}_{1} u_{1}^{\prime} \boldsymbol{R}_{2} u_{2}^{\prime}\right\rangle=1
$$

which can be written as

$$
\mathrm{g}_{11}\left(u_{1}^{\prime}\right)^{2}+2 \mathrm{~g}_{12} u_{1}^{\prime} u_{2}^{\prime}+\mathrm{g}_{22}\left(u_{2}^{\prime}\right)^{2}=1
$$

Substituting (3.4) into (3.6) yields

$$
\begin{aligned}
& u_{1}^{\prime}=h_{2}\left(\left(h_{2}\right)^{2} \mathrm{~g}_{11}-2 h_{1} h_{2} g_{12}+\left(h_{1}\right)^{2} g_{22}\right)^{-\frac{1}{2}}, \\
& u_{2}^{\prime}=-h_{1}\left(\left(h_{2}\right)^{2} \mathrm{~g}_{11}-2 h_{1} h_{2} g_{12}+\left(h_{1}\right)^{2} g_{22}\right)^{-\frac{1}{2}} .
\end{aligned}
$$

The unit tangent vector field of the intersection curve is given by substituting (3.7) into (2.12) as follows

$$
\boldsymbol{t}=\frac{\zeta}{\|\zeta\|} ; \quad \zeta=h_{2} \boldsymbol{R}_{1}-h_{1} \boldsymbol{R}_{2}
$$

\subsection{Curvature and Curvature Vector}

The curvature vector is given by differentiation (3.8) with respect to $\mathrm{s}$ as follows

$$
\begin{aligned}
\boldsymbol{\alpha}^{\prime \prime} & =\frac{\|\zeta\|^{2} \boldsymbol{\zeta}^{\prime}-\left\langle\zeta^{\prime}, \zeta\right\rangle \zeta}{\|\zeta\|^{3}} \\
\boldsymbol{\zeta}^{\prime} & =\|\zeta\|^{-1}\left(\left(h_{2}\right)^{2} \boldsymbol{R}_{11}+\left(h_{1}\right)^{2} \boldsymbol{R}_{22}-2 h_{1} h_{2} \boldsymbol{R}_{12}\right. \\
& \left.+\left(h_{2} h_{12}-h_{1} h_{22}\right) \boldsymbol{R}_{1}+\left(h_{1} h_{12}-h_{2} h_{11}\right) \boldsymbol{R}_{2}\right)
\end{aligned}
$$

The unit principal normal vector field, the curvature and the unit binormal vector are given by using (2.3) (2.4) and (2.5) as follows

$$
\begin{aligned}
& \boldsymbol{n}=\frac{\|\zeta\|^{2} \zeta^{\prime}-\left\langle\zeta^{\prime}, \zeta\right\rangle \zeta}{\|\| \zeta\left\|^{2} \zeta^{\prime}-\left\langle\zeta^{\prime}, \zeta\right\rangle \zeta\right\|}, \\
& \kappa=\frac{\|\| \zeta\left\|^{2} \zeta^{\prime}-\left\langle\zeta^{\prime}, \zeta\right\rangle \zeta\right\|}{\|\zeta\|^{3}}, \\
& \boldsymbol{b}=\frac{\zeta}{\|\zeta\|} \times \frac{\|\zeta\|^{2} \zeta^{\prime}-\left\langle\zeta^{\prime}, \zeta\right\rangle \zeta}{\|\| \zeta\left\|^{2} \zeta^{\prime}-\left\langle\zeta^{\prime}, \zeta\right\rangle \zeta\right\|}
\end{aligned}
$$

\subsection{Torsion and Higher-Order Derivatives}

Equation (3.7) can be written as 


$$
u_{1}^{\prime}=\frac{h_{2}}{\|\zeta\|}, \quad u_{2}^{\prime}=\frac{-h_{1}}{\|\zeta\|}
$$

Differentiation (3.13) we obtain

$$
\begin{aligned}
u_{1}^{\prime \prime} & =\left(\frac{h_{12}}{\|\boldsymbol{\zeta}\|}-\frac{\left\langle\boldsymbol{\zeta}^{\prime}, \boldsymbol{\zeta}\right\rangle}{\|\boldsymbol{\zeta}\|^{2}}\right) u_{1}^{\prime}-\frac{h_{22}}{\|\boldsymbol{\zeta}\|} u_{2}^{\prime}, \\
u_{2}^{\prime \prime} & =-\frac{h_{11}}{\|\boldsymbol{\zeta}\|} u_{1}^{\prime}-\left(\frac{h_{12}}{\zeta}+\frac{\left\langle\boldsymbol{\zeta}^{\prime}, \boldsymbol{\zeta}\right\rangle}{\|\zeta\|^{2}}\right) u_{2}^{\prime}, \\
\boldsymbol{\zeta}^{\prime} & =\left(h_{12} \boldsymbol{R}_{1}+h_{2} \boldsymbol{R}_{11}-h_{11} \boldsymbol{R}_{2}-h_{1} \boldsymbol{R}_{12}\right) u_{1}^{\prime} \\
& +\left(h_{22} \boldsymbol{R}_{1}+h_{2} \boldsymbol{R}_{12}-h_{12} \boldsymbol{R}_{2}-h_{1} \boldsymbol{R}_{22}\right) u_{2}^{\prime} .
\end{aligned}
$$

Differentiation (3.12) we obtain

$$
\begin{aligned}
& u_{1}^{\prime \prime \prime}=\left(\frac{h_{12}}{\|\zeta\|}-\frac{\left\langle\zeta^{\prime}, \zeta\right\rangle}{\|\zeta\|^{2}}\right) u_{1}^{\prime \prime}-\frac{h_{22}}{\|\zeta\|} u_{2}^{\prime \prime}+\frac{h_{112}}{\|\zeta\|}\left(u_{1}^{\prime}\right)^{2} \\
& +\left(2 \frac{\left\langle\zeta^{\prime}, \zeta\right\rangle^{2}}{\|\zeta\|^{4}}-\frac{\left\langle\zeta^{\prime}, \zeta\right\rangle h_{12}}{\|\zeta\|^{3}}-\frac{\left\langle\zeta^{\prime \prime}, \zeta\right\rangle}{\|\zeta\|^{2}}-\frac{\left\|\zeta^{\prime}\right\|^{2}}{\|\zeta\|^{2}}\right) u_{1}^{\prime} \\
& +\frac{2 h_{122}}{\|\zeta\|} u_{1}^{\prime} u_{2}^{\prime}+\frac{h_{222}}{\|\zeta\|}\left(u_{2}^{\prime}\right)^{2}+\frac{\left\langle\zeta^{\prime}, \zeta\right\rangle h_{22}}{\|\zeta\|^{3}} u_{2}^{\prime}, \\
& u_{2}^{\prime \prime \prime}=\frac{-h_{11}}{\|\zeta\|} u_{1}^{\prime \prime}-\left(\frac{h_{12}}{\|\zeta\|}+\frac{\left\langle\zeta^{\prime}, \zeta\right\rangle}{\|\zeta\|^{2}}\right) u_{2}^{\prime \prime} \\
& +\frac{h_{111}}{\|\zeta\|}\left(u_{1}^{\prime}\right)^{2}-\frac{h_{122}}{\|\zeta\|}\left(u_{2}^{\prime}\right)^{2}-\frac{\left\langle\zeta^{\prime}, \zeta\right\rangle h_{11}}{\|\zeta\|^{3}} u_{1}^{\prime} \\
& +\left(2 \frac{\left\langle\boldsymbol{\zeta}^{\prime}, \boldsymbol{\zeta}\right\rangle^{2}}{\|\zeta\|^{4}}-\frac{\left\langle\boldsymbol{\zeta}^{\prime}, \boldsymbol{\zeta}\right\rangle h_{12}}{\|\zeta\|^{3}}-\frac{\left\langle\boldsymbol{\zeta}^{\prime \prime}, \boldsymbol{\zeta}\right\rangle}{\|\boldsymbol{\zeta}\|^{2}}-\frac{\left\|\boldsymbol{\zeta}^{\prime}\right\|^{2}}{\|\boldsymbol{\zeta}\|^{2}}\right) u_{2}^{\prime}, \\
& \boldsymbol{\zeta}^{\prime \prime}=u_{1}^{\prime \prime}\left(h_{12} \boldsymbol{R}_{1}+h_{2} \boldsymbol{R}_{11}-h_{11} \boldsymbol{R}_{2}-h_{1} \boldsymbol{R}_{12}\right) \\
& +u_{2}^{\prime \prime}\left(h_{22} \boldsymbol{R}_{1}+h_{2} \boldsymbol{R}_{12}-h_{12} \boldsymbol{R}_{2}-h_{1} \boldsymbol{R}_{22}\right) \\
& +\left(u_{1}^{\prime}\right)^{2}\left(\begin{array}{c}
h_{112} \boldsymbol{R}_{1}+2 h_{12} \boldsymbol{R}_{11}+h_{2} \boldsymbol{R}_{111} \\
-h_{111} \boldsymbol{R}_{2}-2 h_{11} \boldsymbol{R}_{12}-h_{1} \boldsymbol{R}_{112}
\end{array}\right) \\
& +\left(u_{2}^{\prime}\right)^{2}\left(\begin{array}{c}
h_{222} \boldsymbol{R}_{1}+2 h_{22} \boldsymbol{R}_{12}+h_{2} \boldsymbol{R}_{122} \\
-h_{122} \boldsymbol{R}_{2}-2 h_{12} \boldsymbol{R}_{22}-h_{1} \boldsymbol{R}_{222}
\end{array}\right) \\
& +2 u_{1}^{\prime} u_{2}^{\prime}\left(\begin{array}{c}
h_{122} \boldsymbol{R}_{1}-2 h_{112} \boldsymbol{R}_{2}+h_{22} \boldsymbol{R}_{11} \\
-h_{11} \boldsymbol{R}_{22}+2 h_{2} \boldsymbol{R}_{112}-h_{1} \boldsymbol{R}_{122}
\end{array}\right) \text {. }
\end{aligned}
$$

Substituting $u_{1}^{\prime}, u_{1}^{\prime \prime}, u_{1}^{\prime \prime \prime}, u_{2}^{\prime}, u_{2}^{\prime \prime}$ and $u_{2}^{\prime \prime \prime}$ into (2.14) we obtain the third-order derivative vector of the intersection curve. Hence the torsion can be obtained by (2.7).

We can compute all higher-order derivatives of the intersection curve by a similar way.

\section{Tangentially Intersection Curves}

Assume that the surfaces $f\left(x_{1}, x_{2}, x_{3}\right)=0$ and
$\boldsymbol{R}=R\left(u_{1}, u_{2}\right) ; c_{1}<u_{1}<c_{2}, c_{3}<u_{2}<c_{4}$ are intersecting tangentially at a point $P$ on the curve (3.2) then the unit surface normal vector field of both surfaces are parallel to each other. In other words

$$
\frac{\nabla \boldsymbol{f}}{\|\nabla \boldsymbol{f}\|}= \pm \frac{\boldsymbol{R}_{1} \times \boldsymbol{R}_{2}}{\left\|\boldsymbol{R}_{1} \times \boldsymbol{R}_{2}\right\|}
$$

which can be written as

$$
\nabla \boldsymbol{f}=A\left(\boldsymbol{R}_{1} \times \boldsymbol{R}_{2}\right), A= \pm \frac{\|\nabla \boldsymbol{f}\|}{\left\|\boldsymbol{R}_{1} \times \boldsymbol{R}_{2}\right\|}
$$

Then we can write

$$
\begin{aligned}
& \boldsymbol{f}_{1}=A\left(\boldsymbol{R}_{1}^{2} \boldsymbol{R}_{2}^{3}-\boldsymbol{R}_{1}^{3} \boldsymbol{R}_{2}^{2}\right), \\
& \boldsymbol{f}_{2}=A\left(\boldsymbol{R}_{1}^{3} \boldsymbol{R}_{2}^{1}-\boldsymbol{R}_{1}^{1} \boldsymbol{R}_{2}^{3}\right), \\
& \boldsymbol{f}_{3}=A\left(\boldsymbol{R}_{1}^{1} \boldsymbol{R}_{2}^{2}-\boldsymbol{R}_{1}^{2} \boldsymbol{R}_{2}^{1}\right) .
\end{aligned}
$$

Since $\boldsymbol{x}_{i}(s)=\boldsymbol{R}^{i}\left(u_{1}(s), u_{2}(s)\right), i=1,2,3$, then we have

$$
\boldsymbol{x}_{i}^{\prime}=\boldsymbol{R}_{1}^{i} u_{1}^{\prime}+\boldsymbol{R}_{2}^{i} u_{2}^{\prime}
$$

\subsection{Tangential Direction}

Projecting the curvature vector $\alpha^{\prime \prime}$ onto the two unit normal vectors of both surfaces yields

$$
\left\langle\boldsymbol{\alpha}^{\prime \prime}, \frac{\nabla \boldsymbol{f}}{\|\nabla \boldsymbol{f}\|}\right\rangle= \pm\left\langle\boldsymbol{\alpha}^{\prime \prime}, \frac{\boldsymbol{R}_{1} \times \boldsymbol{R}_{2}}{\left\|\boldsymbol{R}_{1} \times \boldsymbol{R}_{2}\right\|}\right\rangle
$$

Using (2.15) (2.21) and (4.4) we obtain

$$
\begin{aligned}
& \left(\begin{array}{l}
f_{11}\left(x_{1}^{\prime}\right)^{2}+f_{22}\left(x_{2}^{\prime}\right)^{2}+f_{33}\left(x_{3}^{\prime}\right)^{2} \\
+2\left(f_{12} x_{1}^{\prime} x_{2}^{\prime}+\mathrm{f}_{13} x_{1}^{\prime} x_{3}^{\prime}+\mathrm{f}_{23} x_{2}^{\prime} x_{3}^{\prime}\right)
\end{array}\right) \\
& =-A\left\|\boldsymbol{R}_{1} \times \boldsymbol{R}_{2}\right\|\left(L_{11}\left(u_{1}^{\prime}\right)^{2}+2 L_{12} u_{1}^{\prime} \mathrm{u}_{2}^{\prime}+L_{22}\left(u_{2}^{\prime}\right)^{2}\right)
\end{aligned}
$$

Substituting (4.3) into (4.5) yields

$$
a_{11}\left(\frac{u_{1}^{\prime}}{u_{2}^{\prime}}\right)^{2}+2 \mathrm{a}_{12}\left(\frac{u_{1}^{\prime}}{u_{2}^{\prime}}\right)+\mathrm{a}_{22}=0, u_{2}^{\prime} \neq 0
$$

where

$$
\begin{aligned}
a_{11} & =A \boldsymbol{R}_{1} \times \boldsymbol{R}_{2} L_{11}+f_{11}\left(\boldsymbol{R}_{1}^{1}\right)^{2}+f_{22}\left(\boldsymbol{R}_{1}^{2}\right)^{2} \\
& +f_{33}\left(\boldsymbol{R}_{1}^{3}\right)^{2}+2\left(f_{12} \boldsymbol{R}_{1}^{1} \boldsymbol{R}_{1}^{2}+f_{23} \boldsymbol{R}_{1}^{2} \boldsymbol{R}_{1}^{3}+f_{13} \boldsymbol{R}_{1}^{1} \boldsymbol{R}_{1}^{3}\right), \\
a_{22} & =A \boldsymbol{R}_{1} \times \boldsymbol{R}_{2} L_{22}+f_{11}\left(\boldsymbol{R}_{2}^{1}\right)^{2}+f_{22}\left(\boldsymbol{R}_{2}^{2}\right)^{2} \\
& +f_{33}\left(\boldsymbol{R}_{2}^{3}\right)^{2}+2\left(f_{12} \boldsymbol{R}_{2}^{1} \boldsymbol{R}_{2}^{2}+f_{23} \boldsymbol{R}_{2}^{2} \boldsymbol{R}_{2}^{3}+f_{13} \boldsymbol{R}_{2}^{1} \boldsymbol{R}_{2}^{3}\right), \\
a_{12} & =A \boldsymbol{R}_{1} \times \boldsymbol{R}_{2} L_{12}+f_{11} \boldsymbol{R}_{1}^{1} \boldsymbol{R}_{2}^{1}+f_{22} \boldsymbol{R}_{1}^{2} \boldsymbol{R}_{2}^{2} \\
& +f_{33} \boldsymbol{R}_{1}^{3} \boldsymbol{R}_{2}^{3}+f_{12}\left(\boldsymbol{R}_{1}^{1} \boldsymbol{R}_{2}^{2}+\boldsymbol{R}_{1}^{2} \boldsymbol{R}_{2}^{1}\right) \\
& +f_{23}\left(\boldsymbol{R}_{1}^{2} \boldsymbol{R}_{2}^{3}+\boldsymbol{R}_{1}^{3} \boldsymbol{R}_{2}^{2}\right)+\mathrm{f}_{13}\left(\boldsymbol{R}_{1}^{1} \boldsymbol{R}_{2}^{3}+\boldsymbol{R}_{1}^{3} \boldsymbol{R}_{2}^{1}\right) .
\end{aligned}
$$


This can be written in a matrix form as follows

$$
\boldsymbol{a}_{i j}=\nabla \boldsymbol{f} \boldsymbol{R}_{i j}+\boldsymbol{R}_{i}^{\mathrm{T}} \boldsymbol{H} \boldsymbol{R}_{j}
$$

where $\nabla \boldsymbol{f}=\left[\begin{array}{lll}f_{1} & f_{2} & f_{3}\end{array}\right], \boldsymbol{R}_{i j}=\left[\begin{array}{lll}R_{i j}^{1} & R_{i j}^{2} & R_{i j}^{3}\end{array}\right]^{\mathrm{T}}$, $\boldsymbol{R}_{i}=\left[\begin{array}{lll}R_{i}^{1} & R_{i}^{2} & R_{i}^{3}\end{array}\right]^{\mathrm{T}}$ and $\boldsymbol{H}=\left[\begin{array}{lll}f_{11} & f_{12} & f_{13} \\ f_{12} & f_{22} & f_{23} \\ f_{13} & f_{23} & f_{33}\end{array}\right]$ is the Hessian matrix of the surface $f$. Solving (4.6) for $\frac{u_{1}^{\prime}}{u_{2}^{\prime}}$ yields

$$
u_{1}^{\prime}=B u_{2}^{\prime}, \quad B=\frac{-a_{12} \pm \sqrt{\left(\mathrm{a}_{12}\right)^{2}-a_{11} a_{22}}}{a_{11}}
$$

Substituting (3.7) and (4.7) into (4.8) we obtain

$$
\begin{aligned}
& u_{1}^{\prime}=B\left(B^{2} g_{11}+2 B g_{12}+g_{22}\right)^{-\frac{1}{2}} \\
& u_{2}^{\prime}=\left(B^{2} g_{11}+2 B g_{12}+g_{22}\right)^{-\frac{1}{2}} .
\end{aligned}
$$

Then the unit tangent vector field of the intersection curve is given by

$$
\boldsymbol{t}=\frac{B \boldsymbol{R}_{1}+\boldsymbol{R}_{2}}{\left\|B \boldsymbol{R}_{1}+\boldsymbol{R}_{2}\right\|}
$$

From the previous formulas, it is easy to see that, there are four distinct cases for the solution of (4.6) depending upon the discriminant $\Delta=\left(a_{12}\right)^{2}-a_{11} a_{22}$, these cases are as the following [1]

Lemma 1. The point $P$ is a branch point of the intersection curve (3.2) if $\Delta>0$ and there is another intersection branch crossing the curve (3.2) at that point.

Lemma 2. The surfaces $f$ and $h$ intersect at the point $P$ and at its neighborhood, if $\Delta=0$ and $\left(a_{11}\right)^{2}+\left(a_{12}\right)^{2}+\left(a_{22}\right)^{2} \neq 0$. (Tangential intersection curve).

Lemma 3. The point $P$ is an isolated contact point of the surfaces $f$ and $h$, if $\Delta<0$.

Lemma 4. The surfaces $f$ and $h$ have contact of at least second order at the point $P$, if $a_{11}=a_{12}=a_{22}=0$. (Higher-order contact point).

\subsection{Curvature and Curvature Vector}

Differentiation (4.6) and using (4.9) we obtain

$$
\begin{gathered}
u_{1}^{\prime \prime}-B u_{2}^{\prime \prime}=a_{1}, \\
a_{1}=-u_{2}^{\prime} \frac{\left(a_{11}^{\prime} B^{2}+2 a_{12}^{\prime} B+a_{22}^{\prime}\right)}{\left(a_{11} B+a_{12}\right)} ; \quad a_{11} B+a_{12} \neq 0,
\end{gathered}
$$

where

$$
\begin{array}{r}
a_{i j}^{\prime}=\boldsymbol{t}^{\mathrm{T}} \boldsymbol{H R}_{i j}+\left(\nabla \boldsymbol{f} \boldsymbol{R}_{1 j}+\boldsymbol{R}_{1 i}^{\mathrm{T}} \boldsymbol{H R}_{j}+\boldsymbol{R}_{1}^{\mathrm{T}} \boldsymbol{H R}_{1 j}\right) u_{1}^{\prime} \\
+\left(\nabla \boldsymbol{f} \boldsymbol{R}_{2 i j}+\boldsymbol{R}_{2 i}^{\mathrm{T}} \boldsymbol{H R}_{j}+\boldsymbol{R}_{i}^{\mathrm{T}} \boldsymbol{H}_{2 j}\right) u_{2}^{\prime}+\boldsymbol{R}_{i}^{\mathrm{T}} \boldsymbol{Q} \boldsymbol{R}_{j}, \\
\boldsymbol{Q}=\left[\begin{array}{lll}
\mathrm{H}_{1} & \mathrm{H}_{2} & \mathrm{H}_{3}
\end{array}\right] \boldsymbol{t}, \quad \boldsymbol{H}_{i}=\left[\begin{array}{lll}
f_{11 i} & f_{12 i} & f_{13 i} \\
f_{12 i} & f_{22 i} & f_{23 i} \\
f_{13 i} & f_{23 i} & f_{33 i}
\end{array}\right] .
\end{array}
$$

Since the curvature vector is perpendicular to the tangent vector, then we have $\left\langle\boldsymbol{\alpha}^{\prime}, \boldsymbol{\alpha}^{\prime \prime}\right\rangle=0$. Using (2.12) (2.13) and (4.9) we obtain

$$
a_{2} u_{1}^{\prime \prime}+a_{3} u_{2}^{\prime \prime}=a_{4}
$$

where

$$
\begin{aligned}
a_{2} & =B g_{11}+g_{12}, \quad a_{3}=B g_{12}+g_{22}, \\
a_{4} & =-\left(u_{2}^{\prime}\right)^{2}\left(B^{3}\left\langle\boldsymbol{R}_{11}, \boldsymbol{R}_{1}\right\rangle+2 \mathrm{~B}^{2}\left\langle\boldsymbol{R}_{12}, \boldsymbol{R}_{1}\right\rangle\right. \\
& \left.+2 B\left\langle\boldsymbol{R}_{12}, \boldsymbol{R}_{2}\right\rangle+B\left\langle\boldsymbol{R}_{22}, \boldsymbol{R}_{1}\right\rangle+\left\langle\boldsymbol{R}_{22}, \boldsymbol{R}_{2}\right\rangle+\left\langle\boldsymbol{R}_{11}, \boldsymbol{R}_{2}\right\rangle\right)
\end{aligned}
$$

Solving the linear system (4.11) and (4.13) yields

$$
\begin{gathered}
u_{1}^{\prime \prime}=\frac{a_{3} a_{4}+a_{4} B}{a_{3}+a_{2} B}, \\
u_{2}^{\prime \prime}=\frac{a_{4}-a_{1} a_{2}}{a_{3}+a_{2} \mathrm{~B}}
\end{gathered}
$$

The curvature vector of the intersection curve is obtained by substituting $u_{1}^{\prime}, u_{1}^{\prime \prime}, u_{2}^{\prime}$, and $u_{2}^{\prime \prime}$ into (2.13).

\subsection{Torsion}

If we have a branch point, then we can compute the torsion by taking the limit of the torsion of transversal intersection curve at this point. If we have tangential intersection curve, then we can compute $u_{1}^{\prime \prime \prime}$ and $u_{2}^{\prime \prime \prime}$ by differentiation $u_{1}^{\prime \prime}$ and $u_{2}^{\prime \prime}$. Substituting $u_{1}^{\prime}, u_{1}^{\prime \prime}, u_{1}^{\prime \prime \prime}, u_{2}^{\prime}, u_{2}^{\prime \prime}$, and $u_{2}^{\prime \prime \prime}$ into (2.14) we obtain the third-order derivative vector of the intersection curve. Then we can obtain the torsion by using (2.7).

\section{Examples}

Example 1. Consider the intersection of the implicit and the parametric surfaces

$$
\begin{aligned}
& f=x_{1}^{2}+x_{2}^{2}-9=0, \\
& \boldsymbol{R}=\left(u_{1}, 3 \sin u_{2}, 3 \cos u_{2}\right) ; 0<u_{2}<2 \pi .
\end{aligned}
$$

as shown in Figure 1.

Transversal intersection: Using (3.1) yields

$$
h=u_{1}^{2}-9 \cos ^{2} u_{2}=0
$$




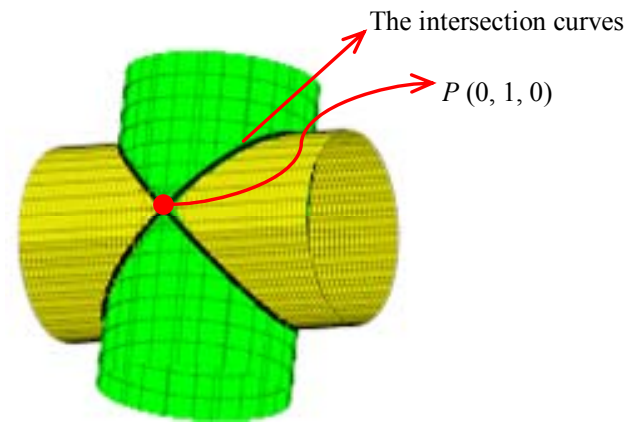

Figure 1. Transversal and tangential intersection.

Differentiation (5.1) and (5.2) we obtain

$$
\begin{aligned}
& h_{1}=2 u_{2}, h_{2}=9 \sin 2 u_{2}, \boldsymbol{R}_{1}=(1,0,0), \\
& h_{22}=18 \cos 2 u_{2}, h_{11}=h_{12}=h_{111}=0, \\
& h_{222}=-36 \sin 2 u_{2}, \\
& \boldsymbol{R}_{1}=(1,0,0), \quad \boldsymbol{R}_{2}=3\left(0, \cos u_{2},-\sin u_{2}\right), \\
& \boldsymbol{R}_{22}=-3\left(0, \sin \sin u_{2}, \cos u_{2}\right), \\
& \boldsymbol{R}_{222}=-3\left(0, \cos \cos u_{2},-\sin u_{2}\right) .
\end{aligned}
$$

Using (3.8) and (5.2), we obtain

$$
\begin{gathered}
\boldsymbol{t}=\left(\frac{\sin u_{2}}{\sqrt{1+\sin ^{2} \mathrm{u}_{2}}}, \frac{-u_{1}}{3 \sqrt{1+\sin ^{2} u_{2}}}, \frac{u_{1} \tan u_{2}}{3 \sqrt{1+\sin ^{2} u_{2}}}\right), \\
\cos u_{2} \neq 0 .
\end{gathered}
$$

Using (3.12) and (5.2), hence

$\zeta=\left(18 \sin u_{2} \cos u_{2},-6 u_{1} \cos u_{2}, 6 u_{1} \sin u_{2}\right)$,

$\zeta^{\prime}=\left(\frac{-2 u_{1} \cos 2 u_{2}}{\cos u_{2} \sqrt{1+\sin ^{2} u_{2}}}, \frac{-6 \sin 2 u_{2}}{\sqrt{1+\sin ^{2} u_{2}}}, \frac{-6 \cos 2 u_{2}}{\sqrt{1+\sin ^{2} u_{2}}}\right)$,

$\|\zeta\|=18 \cos u_{2} \sqrt{1+\sin ^{2} u_{2}}$,

$$
\left\langle\zeta, \zeta^{\prime}\right\rangle=\frac{72 u_{1} \sin ^{3} u_{2}}{\sqrt{1+\sin ^{2} u_{2}}}
$$

Using (2.4), (2.5), (3.12), (3.13) and (5.4) then we have

$$
\begin{aligned}
& \boldsymbol{\alpha}^{\prime \prime}=\left(\frac{-u_{1}}{9\left(1+\sin ^{2} u_{2}\right)^{2}}, \frac{-2 \sin u_{2}}{3\left(1+\sin ^{2} u_{2}\right)^{2}}, \frac{-\cos u_{2}}{3\left(1+\sin ^{2} u_{2}\right)^{2}}\right), \\
& \boldsymbol{n}=\left(\frac{-u_{1}}{3 \sqrt{2} \sqrt{1+\sin ^{2} u_{2}}}, \frac{-\sqrt{2} \sin u_{2}}{\sqrt{1+\sin ^{2} u_{2}}}, \frac{-\cos u_{2}}{\sqrt{2} \sqrt{1+\sin ^{2} u_{2}}}\right), \\
& \kappa=\frac{\sqrt{2}}{3}\left(1+\sin ^{2} u_{2}\right)^{-\frac{3}{2}}, \\
& \boldsymbol{b}=\left(\frac{u_{1}\left(\cos u_{2}+2 \sin u_{2} \tan u_{2}\right)}{3 \sqrt{2}\left(1+\sin ^{2} u_{2}\right)}, 0, \frac{-1}{\sqrt{2}}\right) .
\end{aligned}
$$

Using (3.15) and (3.16) hence

$$
\begin{aligned}
& u_{1}^{\prime}=\frac{\sin u_{2}}{\sqrt{1+\sin ^{2} u_{2}}}, \quad u_{2}^{\prime}=\frac{-u_{1}}{9 \cos u_{2} \sqrt{1+\sin ^{2} u_{2}}}, \\
& u_{1}^{\prime \prime}=\frac{-u_{1}}{9\left(1+\sin ^{2} u_{2}\right)^{2}}, \quad u_{2}^{\prime \prime}=\frac{-\sin u_{2} \cos u_{2}}{9\left(1+\sin ^{2} u_{2}\right)^{2}} .
\end{aligned}
$$

Using (3.17) and (5.7) hence

$$
\begin{aligned}
& u_{1}^{\prime \prime \prime}=\frac{-\sin u_{2}\left(2+3 \cos ^{2} u_{2}\right)}{9\left(1+\sin ^{2} u_{2}\right)^{\frac{7}{2}}}, \\
& u_{2}^{\prime \prime \prime}=\frac{-u_{1}\left(2 \sin u_{2} \tan u_{2}-\cos u_{2} \cos 2 u_{2}\right)}{81\left(1+\sin ^{2} u_{2}\right)^{\frac{7}{2}}} .
\end{aligned}
$$

Using (2.7) and (2.14) yields

$$
\begin{gathered}
\boldsymbol{\alpha}^{\prime \prime \prime}=\left(\frac{-3\left(2+3 \cos ^{2} u_{2}\right) \sin u_{2}}{27\left(1+\sin ^{2} u_{2}\right)^{\frac{7}{2}}, \frac{2 \mathrm{u}_{1}-6 \mathrm{u}_{1} \sin ^{2} u_{2}}{27\left(1+\sin ^{2} u_{2}\right)^{\frac{7}{2}}}}\right) \\
\left.\quad \frac{-\left(4+\sin ^{2} u_{2}\right) \cos u_{2} \sin u_{2}-\left(1+\sin ^{2} u_{2}\right) \sin u_{2}}{27\left(1+\sin ^{2} u_{2}\right)^{\frac{7}{2}}}\right) \\
\tau=\frac{-\sqrt{2}}{4-2 \cos ^{2} u_{2}}\left(4 u_{1} \tan u_{2}-4 \sin u_{2}-10 \cos u_{2} \sin u_{2}\right. \\
+4 \cos ^{2} u_{2} \sin u_{2}+7 \cos ^{3} u_{2} \sin u_{2}-\cos ^{4} u_{2} \sin u_{2} \\
-\cos ^{5} u_{2} \sin u_{2}+2 u_{1} \cos _{2} \sin u_{2}+3 u_{1} \cos ^{3} u_{2} \sin u_{2} \\
\left.+2 u_{1} \cos ^{2} u_{2} \tan u_{2}-6 u_{1} \cos ^{4} u_{2} \tan u_{2}\right)
\end{gathered}
$$

Tangentially intersection: The surfaces are intersecting tangentially at the points $P(0, \pm 1,0)$. Consider the point $P_{1}(0,1,0)$, using (4.7) (4.8) (4.9) and (5.3), then we have

$$
\begin{aligned}
& a_{1}=2, \quad a_{2}=0, \quad a_{3}=-18, \\
& B= \pm 3, \quad u_{1}^{\prime}= \pm \frac{1}{\sqrt{2}}, \quad u_{2}^{\prime}=\frac{1}{3 \sqrt{2}} .
\end{aligned}
$$

Then $\Delta>0$, this means that the point $P_{1}$ is a branch point (Figure 1). From (4.10) and (5.11), we obtain

$$
\boldsymbol{t}=\left( \pm \frac{1}{\sqrt{2}}, 0,-\frac{1}{\sqrt{2}}\right)
$$

Using (2.13) and (4.14) hence 


$$
\begin{aligned}
& u_{1}^{\prime}=u_{2}^{\prime}=0, \quad \boldsymbol{\alpha}^{\prime \prime}=\frac{-1}{6}(0,1,0), \\
& \boldsymbol{n}=(0,-1,0), \quad \kappa=\frac{1}{6}, \\
& \boldsymbol{b}=\left(\frac{-1}{\sqrt{2}}, 0, \mp \frac{1}{\sqrt{2}}\right) .
\end{aligned}
$$

Using (5.10) at $P_{1}(0,1,0)$, we obtain

$$
\begin{aligned}
\tau & =\lim _{u_{2} \rightarrow \frac{\pi}{2}} \frac{-1}{4-2 \cos ^{2} u_{2}}\left(-4 \sqrt{2} \sin u_{2}-\sqrt{2} \cos ^{4} u_{2} \sin u_{2}\right. \\
& +2 \sqrt{2} u_{1} \cos ^{2} u_{2} \tan u_{2}+4 \sqrt{2} \cos ^{2} u_{2} \sin u_{2} \\
& +3 \sqrt{2} u_{1} \cos ^{3} u_{2} \sin u_{2}+7 \sqrt{2} \cos ^{3} u_{2} \sin u_{2} \\
& -10 \sqrt{2} \cos u_{2} \sin u_{2}-\sqrt{2} \cos ^{5} u_{2} \sin u_{2} 4 \sqrt{2} u_{1} \tan u_{2} \\
& \left.+2 \sqrt{2} u_{1} \cos u_{2} \sin u_{2}-6 \sqrt{2} u_{1} \cos ^{4} u_{2} \tan u_{2}\right) \\
& =0
\end{aligned}
$$

Example 2. Consider the intersection of the implicit and the parametric surfaces

$$
\begin{gathered}
f=x_{1}^{2}+x_{2}^{2}+x_{3}^{2}-9=0, \\
\mathrm{R}=\left(u_{1}, 3 \sin u_{2}, 3 \cos u_{2}\right), 0<u_{2}<2 \pi
\end{gathered}
$$

as shown in Figure 2.

At $x_{1}=0,(\nabla \boldsymbol{f}) / /\left(\boldsymbol{R}_{1} \times \boldsymbol{R}_{2}\right)$. Using (4.7) and (5.15), we have $\Delta=0$, this means that the surfaces are intersecting tangentially in a curve as (Figure 2). Then from (4.8) and (4.9), we have

$$
B=0, \quad u_{1}^{\prime}=0, \quad u_{2}^{\prime}=\frac{1}{3}
$$

Using (4.10) we have

$$
\boldsymbol{t}=\left(0, \cos u_{2},-\sin u_{2}\right)
$$

Using (5.16) hence

$$
u_{1}^{\prime \prime}=u_{1}^{\prime \prime \prime}=0, \quad u_{2}^{\prime \prime}=u_{2}^{\prime \prime \prime}=0
$$

Using (2.4) and (2.13) hence the curvature vector and the curvature are given by

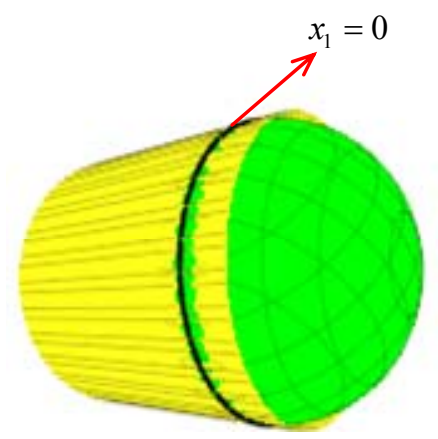

Figure 2. Tangential intersection.

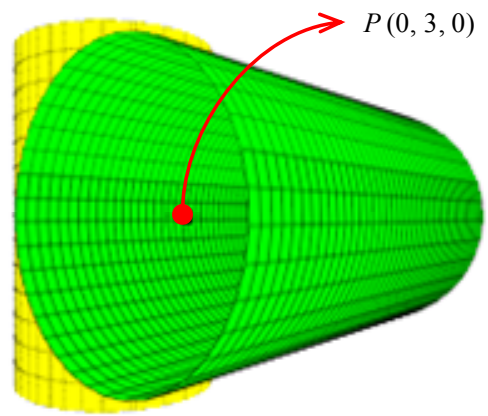

Figure 3. Tangential intersection.

$$
\begin{gathered}
\boldsymbol{\alpha}^{\prime \prime}=-\frac{1}{3}\left(0, \sin u_{2}, \cos u_{2}\right), \\
\boldsymbol{n}=\left(0,-\sin u_{2},-\cos u_{2}\right), \quad \kappa=\frac{1}{3}
\end{gathered}
$$

Using (2.5) (2.7)and (2.14) hence

$$
\begin{aligned}
\boldsymbol{\alpha}^{\prime \prime \prime} & =\frac{1}{9}\left(0,-\cos u_{2}, \sin u_{2}\right), \\
\boldsymbol{b} & =(-1,0,0), \quad \tau=0 .
\end{aligned}
$$

Example 3. Consider the intersection of the implicit and the parametric surfaces

$$
\begin{aligned}
& f=x_{1}^{2}+\left(x_{2}-6\right)^{2}-9=0, \\
& \boldsymbol{R}=\left(u_{1}, 3+3 \sin u_{2}, 3 \cos u_{2}\right) .
\end{aligned}
$$

as shown in Figure 3.

At the point $P(0,3,0),(\nabla \boldsymbol{f}) / /\left(\boldsymbol{R}_{1} \times \boldsymbol{R}_{2}\right)$. Using (4.7) and (5.21), we have $\Delta<0$, this means that the point $P$ is an isolated tangential contact point (Figure 3).

Example 4. Consider the intersection of the implicit and the parametric surfaces

$$
\begin{gathered}
f=x_{3}^{2}-x_{1}^{2}-x_{2}^{2}=0 \\
\boldsymbol{R}=\left(u_{1}\left(1+u_{2}^{2}\right),-u_{2}\left(1+u_{1}^{2}\right), u_{1}^{2}-u_{2}^{3}\right) .
\end{gathered}
$$

as shown in Figure 4.

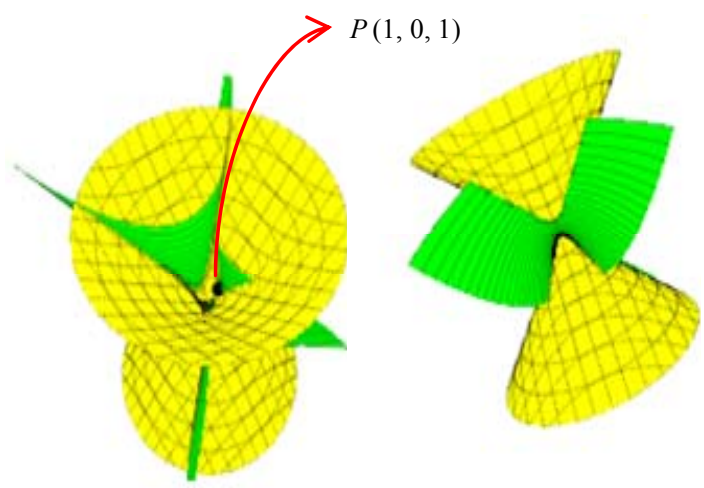

Figure 4. Transversal intersection. 
At the point $P=(1,0,1) \in S^{f} \cap S^{R}$, on the intersection curve (Figure 4), we have

$$
\begin{aligned}
& \boldsymbol{R}_{1}=(1,0,2), \boldsymbol{R}_{11}=(0,0,2), h_{111}=24, \\
& \boldsymbol{R}_{2}=(0,-2,0), \boldsymbol{R}_{22}=(2,0,0), \boldsymbol{R}_{12}=(0,-2,0), \\
& \boldsymbol{R}_{112}=(0,-2,0), \boldsymbol{R}_{122}=(2,0,0), \boldsymbol{R}_{222}=(0,0,-6), \\
& h_{1}=2, h_{11}=10, h_{22}=h_{222}=-12, \\
& h_{2}=h_{12}=0, h_{122}=-24, h_{112}=0 .
\end{aligned}
$$

Using (3.8) and (5.23), we obtain

$$
\boldsymbol{t}=(0,1,0)
$$

Using (3.12) (3.13) and (5.23) we obtain

$$
\begin{gathered}
\boldsymbol{\alpha}^{\prime \prime}=(2,0,3), \\
\boldsymbol{n}=\left(\frac{2}{\sqrt{13}}, 0, \frac{3}{\sqrt{13}}\right), \kappa=\sqrt{13} .
\end{gathered}
$$

Using (2.5) (2.7) (2.14) (3.17) and (5.25) we obtain

$$
\begin{gathered}
\boldsymbol{\alpha}^{\prime \prime \prime}=\left(\frac{-3}{4},-19, \frac{-3}{4}\right), \\
\boldsymbol{b}=\left(\frac{3}{\sqrt{13}}, 0, \frac{-2}{\sqrt{13}}\right), \quad \tau=-\frac{3}{52} .
\end{gathered}
$$

\section{Conclusions}

Algorithms for computing the differential geometry properties of intersection curves of implicit and parametric surfaces in $\mathbb{R}^{3}$ are given for transversal and tangential intersection. This paper is an extension to the works of Ye and Maekawa [1]. They gave algorithms to compute the differential geometry properties of intersection curves between two parametric surfaces then they applied it on a simple example for implicit and parametric surfaces intersection. This paper presented direct and simple formulas to compute all differential geometry properties, which may reduce the time it takes to calculate those properties. The types of singularities on the intersection curve are characterized. The questions of how to exploit and extend these algorithms to compute the differential geometry properties of intersection curves between three surfaces in $\mathbb{R}^{4}$, can be topics of future research.

\section{Acknowledgements}

The authors would like to thank the reviewers for their valuable comments and suggestions.

\section{References}

[1] X. Ye andT. Maekawa, "Differential Geometry of Intersection Curves of Two Surfaces," Computer-Aided Geometric Design, Vol. 16, No. 8, September 1999, pp. $767-$ 788. doi:10.1016/S0167-8396(99)00018-7

[2] C. L. Bajaj, C. M. Hoffmann, J. E. Hopcroft and R. E. Lynch, "Tracing Surface Intersections," Computer-Aided Geometric Design, Vol. 5, No. 4, November 1988, pp. 285-307. doi:10.1016/0167-8396(88)90010-6

[3] N. M. Patrikalakis, "Surface-to-Surface Intersection," IEEE Computer Graphics \& Applications, Vol. 13, No. 1, January-February 1993, pp. 89-95. doi:10.1109/38.180122

[4] T. J. Willmore, "An Introduction to Differential Geometry," Clarendon Press, Oxford, 1959.

[5] M. Düldül, "On the Intersection Curve of Three Parametric Hypersurfaces," Computer-Aided Geometric Design, Vol. 27, No. 1, January 2010, pp. 118-127. doi:10.1016/j.cagd.2009.10.002

[6] E. Kruppa, "Analytische und Konstruktive Differentialgeometrie," Springer, Wien, 1957.

[7] E. Hartmann, "G2 Interpolation and Blending on Surfaces," The Visual Computer, Vol. 12, No. 4, 1996, pp. 181-192. doi: 10.1007/s003710050057

[8] G. A. Kriezis, N. M. Patrikalakis and F.-E. Wolter, "Topological and Differential Equation Methods for Surface Intersections," Computer-Aided Geometric Design, Vol. 24, No. 1, January 1992, pp. 41-55. doi:10.1016/0010-4485(92)90090-W

[9] R. C. Luo, Y. Ma and D. F. McAllister, "Tracing Tangential Surface-Surface Intersections," Proceedings Third ACM Solid Modeling Symposium, Salt Lake City, 1995, pp. 255-262. doi: 10.1145/218013.218070

[10] O. Aléssio, "Differential Geometry of Intersection Curves in $\mathbb{R}^{4}$ of three Implicit Surfaces," Computer-Aided Geometric Design, Vol. 26, No. 4, May 2009, pp. 455471. doi:10.1016/j.cagd.2008.12.001

[11] M. P. do Carmo, "Differential Geometry of Curves and Surface," Prentice Hall, Englewood Cliffs, NJ, 1976.

[12] J. J. Stoker, "Differential Geometry," Wiley, New York, 1969.

[13] D. J. Struik, "Lectures on Classical Differential Geometry," Addison-Wesley, Reading, 1950. 